Relations industrielles

Industrial Relations

\title{
The Strike, For and Against, Harold H. Hart (ed.), New York, Hart Publishing Company Inc., 1971, 249 pp.
}

\section{Jean Boivin}

Volume 28, numéro 3, 1973

URI : https://id.erudit.org/iderudit/028435ar

DOI : https://doi.org/10.7202/028435ar

Aller au sommaire du numéro

Éditeur(s)

Département des relations industrielles de l'Université Laval

ISSN

0034-379X (imprimé)

1703-8138 (numérique)

Découvrir la revue

Citer ce compte rendu

Boivin, J. (1973). Compte rendu de [The Strike, For and Against, Harold H. Hart (ed.), New York, Hart Publishing Company Inc., 1971, 249 pp.] Relations industrielles / Industrial Relations, 28(3), 672-674.

https://doi.org/10.7202/028435ar

Tous droits réservés @ C Département des relations industrielles de l'Universite Laval, 1973
Ce document est protégé par la loi sur le droit d'auteur. L’utilisation des services d'Érudit (y compris la reproduction) est assujettie à sa politique d'utilisation que vous pouvez consulter en ligne.

https://apropos.erudit.org/fr/usagers/politique-dutilisation/ 
au contexte actuel des relations du travail. Signalons à titre d'exemple les commentaires qu'ils formulent notamment sur la question de l'accès des cadres au régime de la négociation collective de même que sur la situation des relations de travail dans le secteur public aux Etats-Unis.

Même si le juriste à l'esprit cartésien risque de ne pas y trouver l'approche traditionnelle qui préside à la rédaction des traités de droit, l'ouvrage pourra lui être d'une utilité certaine grâce à l'abondante jurisprudence qui y est citée. Signalons également que ce livre comporte l'avantage de présenter en annexe la plupart des grands textes de loi qui régissent les rapports collectifs du travail aux Etats-Unis.

Nous croyons que ce livre peut être utilisé avec profit comme manuel d'enseignement pour des étudiants de relations industrielles, de sciences de l'administration ou autres.

\section{Jean BERNIER}

\section{Université Laval}

The Strike, For and Against, Harold $\mathrm{H}$. Hart (ed.), New York, Hart Publishing Company Inc., 1971, 249 pp.

Ce livre est un recueil d'opinions sur la grève. Comme son titre l'indique, le livre contient des opinions favorables ainsi que d'autres défavorables à la grève. Si tous les auteurs sauf un (il s'agit d'un journaliste célèbre pour ses idées anti-syndicales) s'entendent pour admettre que la grève est un moyen de pression légitime pour permettre aux travailleurs d'améliorer leurs conditions de travail dans le secteur privé, l'unanimité est loin d'être faite en ce qui concerne les travailleurs du secteur public.

On peut avoir une assez bonne idée de l'opinion de chaque auteur en lisant la brève biographie qui précède chaque article. Ainsi, les deux syndicalistes, Victor Gotbaum, président de l'American Federation of State, Country and Municipal Employees et Paul Jennings, président de l'International Union of Electrical Workers, s'entendent pour affirmer que le droit de grève est inaliénable même dans le secteur public. Leur pré- occupation est surtout de montrer que les travailleurs ne font pas la grève par plaisir mais que la plupart du temps ils sont poussés à recourir à un arrêt de travail par suite d'une attitude intransigeante ou injuste de l'employeur. Gotbaum et Jennings mentionnent plusieurs de ces grèves où les travailleurs n'avaient pas d'autre choix que la grève.

Il existe d'autre part une catégorie d'opinions que l'on pourrait qualifier de jugements honnêtes sur le problème mais dans lesquelles les auteurs s'évertuent à ménager la chèvre et le chou. On se dit en principe en faveur du droit de grève mais on énumère toute une liste de situations où le droit de grève devrait être retiré. Parmi cette catégorie on retrouve principalement deux journalistes de même qu'un consultant patronal.

De toutes les opinions, il y en a trois qui apportent des considérations plus intéressantes que les autres. L'article de Theodore Kheel et Lewis B. Kadden est parmi ce groupe. Theodore Kheel n'a pas besoin de présentation : il a actuellement la réputation d'être l'un des meilleurs (sinon le meilleur) arbitre et médiateur en relations industrielles aux Etats-Unis. Quant à Lewis B. Kadden, il est un avocat réputé dans l'est des Etats-Unis. Kheel et Kadden contredisent l'opinion fort répandue aux Etats-Unis et dont les principaux défenseurs sont les professeurs Harry $\mathbf{H}$. Wellington et Ralph $\mathbf{K}$. Winter, que l'octroi du droit de grève aux employés du secteur public a pour effet de donner trop de pouvoir aux syndicats. Alors que l'expérience du Québec semble leur donner raison, Kheel et Kadden font cependant reposer leur argumentation sur d'autres facteurs : ils affirment que la négociation collective sans le droit de grève est impossible et que, lorsque les opposants au droit de grève justifient leurs positions, c'est plutôt contre l'institution proprement dite qu'est la négociation collective qu'ils s'attaquent.

Kheel et Kadden avancent plusieurs autres bonnes idées mais on peut leur reprocher de n'être pas très originaux dans leurs solutions aux conflits de travail. En effet, après s'être prononcés en faveur du droit de grève et avoir proposé la création d'une Commission indépendante qui fournirait des médiateurs dans les conflits, ils se contentent de suggérer 
la possibilité d'injonctions 《ad hoc》 pour arrêter une grève qui menacerait des services essentiels. De telles procédures existent déjà au Québec ainsi que dans les Etats de Pennsylvanie et d'Hawaii. Par contre, on peut leur reconnaître d'avoir vu juste quand ils affirment que ces procédures ont des chances d'être efficaces uniquement si elles ne sont pas utilisées fréquemment. L'expérience du Québec en fournit un témoignage assez évident. Enfin, Kheel et Kadden investiraient leur Commission indépendante $\mathrm{du}$ pouvoir de trancher tout litige qui n'aurait pas été réglé après épuisement de la procédure d'injonctions.

Pour sa part, le professeur de philosophie Will Herberg pose très bien le problème du droit de grève dans le secteur public quand il affirme : «On the one hand, it would seem to be impossible, within the limits of constitutionality, to compel citizens not convicted of crime to work against their will, or to refrain from combining to withhold their services; that would be too close to involuntary servitude. On the other hand, recent experience has shown that the unrestricted exercise of this « right to strike 》 may, on occasion, endanger the public order and, in particular, jeopardize the health, welfare and sometimes even the very survival of the community. This is the dilemma 》 ( $p$. 149).

Ensuite, Herberg affirme qu'une distinction fondamentale doit être établie entre les fonctions et activités du gouvernement qui sont l'expression de sa souveraineté et celles qui ne le sont pas. En ce qui concerne les premières, il s'agit d'activités dans lesquelles aucune grève ne peut être tolérée car elles pourraient mettre en danger la société. Ces activités, selon Herberg, sont les diverses composantes de la défense nationale telles la marine, l'armée et l'aviation, le service postal ainsi que les services de police et de protection contre le feu. Alors qu'Herberg retire à ces employés le droit de faire la grève il mentionne par contre très judicieusement que ceux-ci doivent être adéquatement rétribués pour la perte de ces droits qui sont reconnus aux autres employés.

Herberg s'attaque ensuite aux législations comme le Taylor Act de l'Etat de New York qui interdisent sans distinc- tion entre les différents services le droit de grève aux employés du secteur public. Herberg suggère que le droit de grève devrait être reconnu à tous les autres employés qui ne travaillent pas dans les services où la souveraineté gouvernementale peut être impliquée. Quant aux situations où l'intérêt public peut être affecté comme dans le cas des hôpitaux et des chemins de fer (un secteur vital pour le transport des denrées alimentaires aux Etats-Unis), Herberg propose un système de médiation, suivi d'une période de "cooling-off» et, en dernier ressort, l'arbitrage obligatoire.

Dans le dernier article du livre, Carey McWilliams, éditeur de la revue The Nation, présente un point de vue très sensé en faveur du droit de grève dans le secteur public. Même si son argumentation se situe surtout au niveau des principes, il en ressort néanmoins un bon nombre de considérations intéressantes. D'abord il affirme que les grèves fournissent une excellente illustration de la tendance généralisée parmi l'opinion publique et les gouvernements de s'intéresser davantage aux symptômes et aux conséquences des problèmes plutôt qu'à leurs causes. Selon McWilliams, les grèves ne sont que des symptômes et non des causes de sorte qu'en imposant des restrictions au droit de grève on évite les vrais problèmes.

Une autre observation très judicieuse est la citation de John 'T. Dunlop qu'utilise McWilliams à la fin de son article: «There can be no single standardized formula for settling what are called emergency disputes... If mediation fails, then what? If the leaders are put in jail and there is still no settlement, then what?》 (p. 225). McWilliams croit que chaque grève doit être jugée à son mérite et il s'objecte à ce qu'une législation générale règlemente minitieusement l'exercice du droit de grève, car «a single legislative prescription for all situations, including those that may conceivably strike in the future, will inevitably be ill adapted to many of them》 (p. 227).

La conclusion de McWilliams devrait être lue et analysée attentivement par certains gouvernements qui s'apprêtent à légiférer sur le droit de grève : «Strikes are part of the price we pay for what we term a free society. All things con- 
sidered, it is not an exorbitant price. If we attempt to suppress strikes, we will find ourselves drifting in the direction of a dictatorial regime. The best prescription for strikes is to see to it that labor - and other groups - are not forced to strike.

\section{Jean BOIVIN}

\section{Université Laval}

Management Decisions and Behavior, by Max D. Richards and Paul S. Greenlaw, Georgetown, Ontario, Irwin-Dorsey Limited, 1972, 655 pp.

Ce manuel revisé d'introduction à l'administration demeure fidèle à la version originale parue en 1966 quant au cadre conceptuel et au contenu. Cependant, pour chacun de ces deux points, les auteurs ont mieux précisé leur approche en complétant par la notion de système le point de vue selon lequel ils abordent l'administration, soit celui de la décision tel que Simon le proposait. Ils élargissent ainsi leur cadre théorique initial.

Cependant, l'effort de renouvellement du manuel ne se détache pas de l'Ecole classique en administration. A cet égard, la notion de système est appliquée à l'organisation en tant que telle. Aussi, l'administration est présentée comme un soussystème quasi spécifique d'un ensemble plus vaste, ou de l'environnement. Mais les auteurs ne prennent pas en considération ce dernier concept d'une manière systématique dans un manuel. Pour y parvenir, ils essaient dans un effort ultime de faire appel au travail de Lawrence et Lorsch (Organization and Environment) mais ils oublient que l'environnement n'est traité par ces auteurs que du point de vue de ceux qui ont à prendre des décisions, et ne constitue pas un objet d'analyse spécifique. Le concept d'environnement demeure général et abstrait dans le manuel.

En ce sens, lorsque les auteurs essaient d'énoncer une théorie de l'administration en termes de «situation》 ou de «management conditionnel», ils demeurent prisonniers de leur méthode d'approche. Ils ne s'éloignent pas pour la peine des modèles d'analyse énoncés par Burns et Stalker (The Management of Innovation) dans leur analyse com- parative d'entreprises industrielles, et de Fiedler (A Theory of Leadership Effectiveness) pour l'analyse du leadership. En outre, dans ce dernier cas, la valeur de l'approche est loin d'être démontrée comme G. Strauss (Organizational Behavior and Personnel Relations. Reprint No 346, Calif. Institute of Industrial Relations, 1970), l'a fait remarquer à juste titre.

D'une manière plus générale, on pourrait dire que les auteurs prennerit trop facilement certaines conclusions de recherche pour définitivement acquises à propos desquelles les chercheurs concernés s'interrogent eux-mêmes. C'est surtout le cas pour tout le contenu des chapitres concernant l'individu, le leadership et le groupe. La limite des théories présentées de même que leurs implications ne sont pas discutées. A ce propos, la question est la suivante : jusqu'à quel point peut-on parler de l'individu en tant qu'individu ou en tant que membre d'un groupe (informel ou autre) sans tenir compte de la relation d'autorité où il est placé ? Si on ne le fait pas, l'aspect structurel de l'intégration de l'individu à l'organisation n'est pas traité, et bien sûr, la notion de conflit est évitée ou ignorée dans l'analyse.

La même faiblesse se reproduit lorsqu'il s'agit d'examiner les processus organisationnels au chapitre 8 . Les auteurs donnent l'une à la suite de l'autre deux thèses explicatives de la structure administrative, l'une fondée sur la stratégie administrative, l'autre sur la technologie. Bien que ces thèses soient aux antipodes l'une de l'autre, les auteurs ne s'en souviennent guère. Par ailleurs, les recherches ayant démontré que la structure administrative n'est pas dépendante de la technologie, et dont certaines sont parues vers le milieu des années ' 60 ne sont pas mentionnées.

Lorsqu'il s'agit d'énoncer des principes classiques du management ou techniques administratives les auteurs sont irréprochables, et demeurent fidèles à l'Ecole classique. Par contre, c'est justement l'utilisation de cette même démarche intellectuelle pour l'analyse de l'organisation administrative du point de vue des sciences du comportement, qui constitue la faiblesse majeure du manuel.

Etant donné nos réserves, si l'enseignement universitaire conserve la formation 